\title{
THE EFFECT OF RETICULOCYTE HEMOGLOBIN CONTENT ON THE DIAGNOSIS OF IRON DEFICIENCY ANEMIA: A META-ANALYSIS STUDY
}

\author{
EFEKAT SADRŽAJA RETIKULOCITNOG HEMOGLOBINA NA DIJAGNOSTIKOVANJE \\ ANEMIJE USLED NEDOSTATKA GVOŽĐA: META-ANALIZA
}

\author{
Merve Kılıç ${ }^{1,2}$, Aysel Özpınar ${ }^{1}$, Mustafa Serteser ${ }^{1}$, Meltem Kilercik¹, Muhittin Serdar ${ }^{1}$ \\ ${ }^{1}$ Acıbadem Mehmet Ali Aydinlar University, School of Medicine, Dept. Medical Biochemistry, Istanbul, Turkey \\ ${ }^{2}$ Dogus University, School of Vocational, Dept. Anesthesia Program, Istanbul, Turkey
}

\begin{abstract}
Summary
Background: Iron deficiency anemia (IDA) is the most common type of anemia worldwide and has many adverse effects on life quality. This meta-analysis study aims to show that reticulocyte hemoglobin content $(\mathrm{CHr})$ is more effective than routinely used parameters in the diagnosis of IDA.

Methods: Comprehensive and systematic research was done using international databases including PubMed, Web of Science, Cochrane Library, Science Direct, and Google Scholar, which contain all articles published on IDA until December 29, 2020. Seventeen articles were included in the meta-analysis.

Results: The analyses found the Cohen's deffect size (Standardized Mean Difference) values of the parameters. Accordingly, $\mathrm{CHr}$ is $2.84(95 \% \mathrm{Cl} 2.36$ to 3.31), mean corpus volume (MCV) is $2.46(95 \% \mathrm{Cl} 1.97$ to 2.95$)$, ferritin is $2.37(95 \% \mathrm{Cl} 1.63$ to 3.11$)$, and transferrin saturation (TSAT) is $3.76(95 \% \mathrm{Cl} 2.14$ to 5.38$)$. To diagnose IDA, the sensitivity value of the $\mathrm{CHr}$ concentration was found as $83.5 \%$ (95\% Cl 76.1 to 89.8$)$, specificity value to be $91.8 \%$ (95\% Cl 85.5 to 96.4 ), and mean cut-off value as $28.2 \mathrm{pg}$. Conclusions: The results of our study reveal the findings that $\mathrm{CHr}$ is a better biomarker than MCV and ferritin used in determining IDA, and its efficacy is lower than TSAT. It is very important to use it routinely for the pre-diagnosis of IDA, which is very important for public health. The groups in the study are heterogeneous but contain bias. Therefore, metaanalyses of studies with less heterogeneity of $\mathrm{CHr}$ are needed.
\end{abstract}

\section{Kratak sadržaj}

Uvod: Anemija usled nedostatka grožđa (IDA) je najčešća vrsta anemije na svetu i ima mnogo negativnih efekata na kvalitet života. Cilj ove meta-analize je da pokaže da je koncentracija retikulocitnog hemoglobina ( $\mathrm{CHr}$ ) efikasnija od rutinski korišćenih parametara u dijagnozi IDA.

Metode: Sprovedeno je sveobuhvatno i sistematično istraživanje gde su upotrebljene međunarodne baze podataka, uključujući PubMed, Web of Science, Cochrane Library, Science Direct i Google Scholar, koje sadrže sve članke objavljene na temu IDA do 29. decembra 2020. Meta-analiza je obuhvatila sedamnaest članaka.

Rezultati: Analizom su utvrđene vrednosti parametara za veličinu efekta Koenovog d (standardizovana srednja razlika). Shodno tome, $\mathrm{CHr}$ je 2,84 (95\% Cl 2,36 do 3,31), srednja zapremina tela (MCV) je 2,46 (95\% Cl 1,97 do 2,95), feritin je 2,37 (95\% Cl 1,63 do 3,11), a zasićenje transferinom (TSAT) je 3,76 (95\% Cl 2,14 do 5,38). Da bi se dijagnostikovala IDA, utvrđena je vrednost osetljivosti koncentracije $\mathrm{CHr}$ na 83,5\% (95\% Cl 76,1 do 89,8), vrednost specifičnosti $91,8 \%(95 \% \mathrm{Cl} 85,5$ do 96,4$)$, a srednja granična vrednost je $28,2 \mathrm{pg}$.

Zaključak: Rezultati naše studije ukazuju da je $\mathrm{CHr}$ bolji biomarker od MCV i feritina koji se koriste u određivanju IDA, a njegova efikasnost je niža od TSAT. Veoma je važno da se rutinski koristi za preddijagnozu IDA, što je veoma važno za javno zdravlje. Grupe u studiji su bile heterogene, ali imaju elemente pristrasnosti, pa je potrebno sprovesti meta-analize sa manjom heterogenosti $\mathrm{CHr}$.

\footnotetext{
Address for correspondence:

Merve Kılıç

Dogus University, School of Vocational, Dept. Anesthesia Program

Dudullu Campus Dudullu Osb Mah. NatoYolu Cad. 265/1,

34775 Ümraniye/Istanbul, Turkey

Phone: +90-444-7997-1959

e-mail: mkilic@dogus.edu.tr
} 
Keywords: Reticulocyte Hemoglobin Content, Iron Deficiency Anemia, Transferrin saturation, Mean Corpus Volume, Ferritin, Meta-Analysis

\section{Introduction}

Iron is an element that has essential functions for human life. While it is found in the structure of hemoglobin $(\mathrm{Hb})$, which provides oxygen transport in the body, it also ensures the fulfilment of iron-related functions by joining the structure of enzyme systems in some tissues (1). Iron deficiency anemia (IDA) occurs when the iron intake in humans is less than its excretion, in other words, when a negative iron balance occurs in the body (increased need for iron, absorption disorders, chronic blood loss) as a result of insufficient iron for $\mathrm{Hb}$ synthesis in the stores $(1,2)$. IDA is the most common type of anemia, and it constitutes the most advanced stage of iron deficiency. The World Health Organization (WHO) describes a hemoglobin value of $<130.0 \mathrm{~g} / \mathrm{L}$ in men, $<120.0$ $\mathrm{g} / \mathrm{L}$ in women, and $<110.0 \mathrm{~g} / \mathrm{L}$ in pregnant women as anemia $(3,4)$. IDA is more common in women than men due to conditions such as menstruation and pregnancy. Adolescence causes an increase in blood pressure due to rapid growth and development and insufficient iron stores. If this condition cannot be compensated, IDA may occur as a result of insufficient intake. The most important reasons for IDA in postmenopausal women and men are the formation and increase of gastrointestinal system (GIS) bleeding (5-7). IDA is characterized by hypochromia and microcytosis in erythrocytes, decreased serum ferritin and serum iron levels, TSAT, and increased total ironbinding capacity $(4,8)$. Low serum ferritin level in IDA is essential and should not always be associated with IDA. Again, because it is an acute phase reactant, its normal condition does not exclude IDA; the underlying etiology must be defined and regulated (9). In contrast to all these conditions, iron overload reduces the efficiency of iron utilization and induces oxidative stress formation (10). In addition to these, free erythrocyte zinc protoporphyrin (ER-ZPP), soluble transferrin receptor (sTfR), and reticulocyte hemoglobin content $(\mathrm{CHr}$ or Ret-He) are among the reliable laboratory test parameters used to describe IDA. Soluble transferrin receptor with increasing erythrocyte ERZPP value causes early deterioration of iron condition and emergence of IDA (11-14).

Bone marrow erythropoietic activity and intracellular iron requirement are important criteria in determining sTfR level. Therefore, in conditions associated with iron deficiency and induced erythropoiesis (sickle cell anemia, megaloblastic anemia, thalassemia, polycythemia, etc.), sTfR concentration increases, while aplastic anemia decreases $(15,16)$. Normal serum sTfR level is $3.5-8.5 \mathrm{mg} / \mathrm{L}$. It is known that a
Ključne reči: Sadržaj retikulocitnog hemoglobina, anemija usled nedostatka gvožđa, zasićenje transferina, srednja zapremina tela, feritin, meta-analiza

high sTfR (>8.5 mg/L) level is an early and sensitive biomarker for the diagnosis of IDA (11).

The ratio of sTfR concentration to logarithmic ferritin level is also determinant in the differential diagnosis of IDA. A ratio of less than 1 is associated with chronic disease anemia, while the ratio higher than 2 is evaluated in favour of IDA (17).

The decrease in iron concentration increases zinc transport in the intestines, and therefore the increased concentration of ER-ZPP $(80 \mu \mathrm{g} / \mathrm{dL})$ in erythrocytes is associated with iron deficiency. However, routine use of ER-ZPP measurements is difficult and time-consuming due to automation difficulties (18).

$\mathrm{CHr}$, also known as Ret-He, measures the amount of hemoglobin in reticulocytes and is an indicator of cell hemoglobination, reflecting the quality of newly produced reticulocytes. Microcytic, hypochromic red blood cell (RBC) is formed due to ongoing reticulocyte production when there is not enough iron. Thus, RET-He reflects an earlier measure of reduced hemoglobin status compared to hemoglobin and hematocrit $(11,19)$.

Reticulocytes are separated from the erythroblasts after $\mathrm{Hb}$ synthesis, pass into the peripheral blood and turn into mature erythrocytes within a few days. Therefore, $\mathrm{CHr}$ is the ideal parameter to be considered for real-time $\mathrm{Hb}$ synthesis. Reticulocyte hemoglobin content is affected only by the amount of iron unless there are hematopoietic disorders (10).

Determination of iron status is possible with RET-He measurement. RET-He is determined by automated fluorescence flow cytometry, which measures the mean values of the forward light scattering intensity of mature red blood cells and reticulocytes using a polymethine dye. The values obtained reflect the reticulocyte hemoglobin content (20). Reticulocyte hemoglobin content is more effective in diagnosing iron deficiency, determining early iron deficiency anemia, differentiation of beta-thalassemia feature, and more effective than the other parameters involved in iron metabolism $(10,12)$. $\mathrm{CHr}$ is a less variable parameter that performs better than ferritin in response to intravenous (IV) iron therapy, providing better diagnostic accuracy for iron $(15,16)$.

In the United States and Europe, $\mathrm{CHr}$ has been accepted as a marker in iron deficiency with a diagnostic threshold of $29 \mathrm{pg}$. However, there is no reference value agreed on the best value for its sensitivity and specificity (17). 
This study examined the effectiveness of $\mathrm{CHr}$ in addition to routine parameters in determining IDA.

\section{Methods}

\section{Literature Search Strategy}

Comprehensive systematic research was carried out using international databases, including PubMed, Web of Science, Cochrane Library, Science Direct, and Google Scholar, to determine all studies on $\mathrm{CHr}$ as a biomarker of IDA.

Our study included all articles published until December 29, 2020. The words used as search criteria in this study are as follows: „Reticulocyte hemoglobin AND iron deficiency anemia OR iron deficiency", »reticulocyte hemoglobin « $O R$ »iron deficiency" $O R$ »iron deficiency anemia«.

This systematic review and meta-analysis were carried out under the guidelines for Preferred Reporting Items for Systematic Reviews and MetaAnalyses (PRISMA).

\section{Selection Criteria}

The full texts of the articles related to the study were examined in terms of exclusion and inclusion criteria. Appropriate studies including clinical features of patients diagnosed with IDA and $\mathrm{CHr}$ used as a biomarker in the diagnosis of the disease were included in the meta-analysis.

Prospective/retrospective cohort, case-control, and cross-sectional studies were found suitable for inclusion in our study.

Repeated articles, reviews, case reports, expert opinions, letters, editorials, studies on experimental animals, studies not including control groups, studies where $\mathrm{CHr}$ is a biomarker but not related to iron deficiency, studies without available data, studies on results other than mean and standard deviation values, articles published in languages other than English, studies involving chronic patient groups with IDA, and studies only on iron deficiency were excluded (Figure 1).

\section{Data Extraction and Quality Assessment}

Data extraction, evaluation of literature quality, and evaluation of bias risk were carried out independently by two researchers (Serdar M. and Kılıç M.).

The following features were extracted: Article information (first author, year of publication), country, study designs, gender, device information, study example, patient/control information, the total number of samples, researched parameters. This informa-

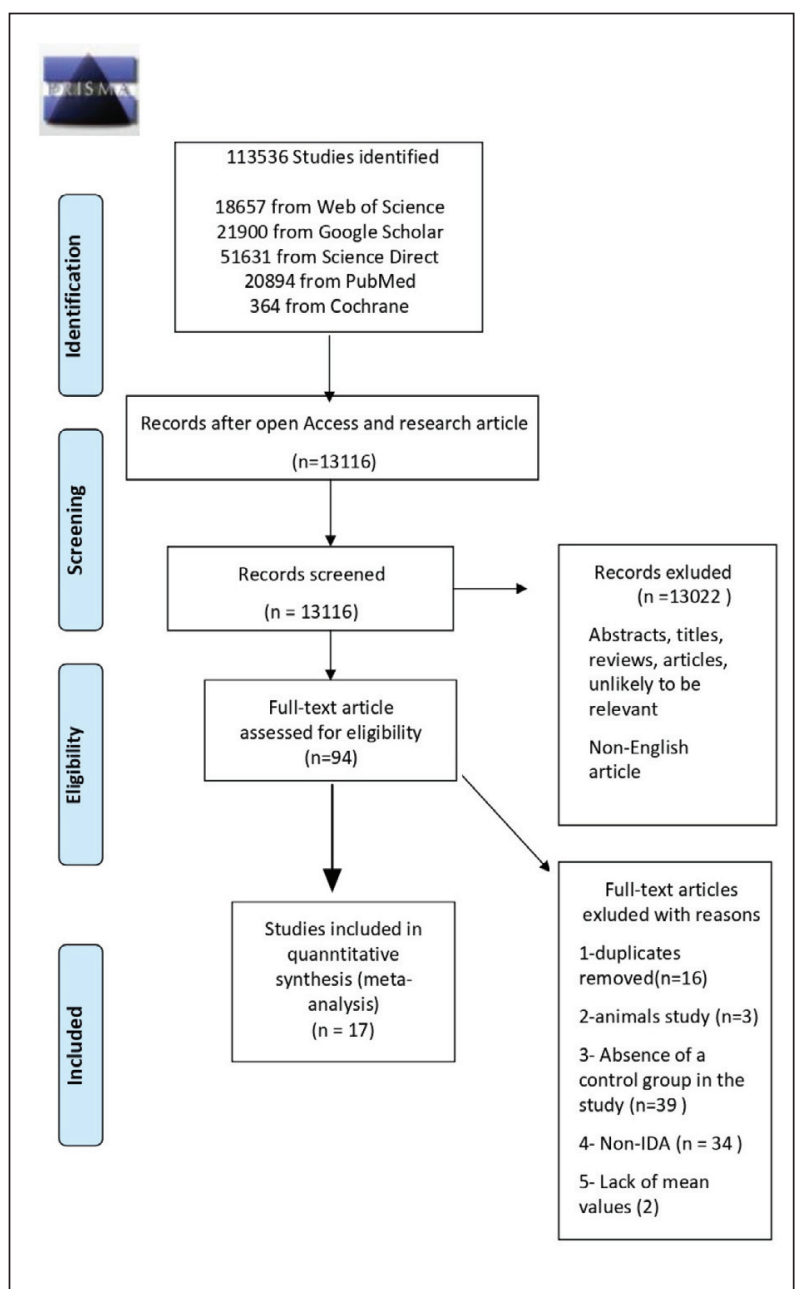

Figure 1 PRISMA flow diagram for inclusion of studies in this meta-analysis.

tion is shown in Table I. Microsoft Excel database was used to save the necessary information.

\section{Statistical Analysis}

The meta-analysis study was performed using MedCalc statistical software, version 19.0.7 (MedCalc Software, Ostend, Belgium). A meta-analysis was performed on Cohen's d effect size (Standardized Mean Difference) by taking the mean and standard deviation values. In the evaluation, the results obtained with the random-effects model of the studies with low bias risk were taken into consideration. The study aimed to perform group analysis of $\mathrm{CHr}$, $M C V$, ferritin, and TSAT biomarkers according to the target range in IDA. Using Cochran's Q statistic and $1^{2}$ statistic respectively, statistical heterogeneity was evaluated. If the $\mathrm{I}^{2}$ statistical value was above 50 per cent and the p-value was lower than 0.05, the heterogeneity was considered statistically significant. 
Table I Features of the studies analyzed for iron deficiency anemia, Sysmex XN Aplpha/10/300/1000, XE 2100/5000 (Sysmex Corporation Kobe, Japan), Technicon H3, (Bayer, Germany), Advia 120, ADVIA 2120i (Siemens AG, Erlangen, Germany).

\begin{tabular}{|c|c|c|c|c|c|c|c|c|c|c|}
\hline FIRST AUTHOR & YEAR & COUNTRY & DEVICE & $\begin{array}{l}\text { STUDY } \\
\text { DESIGN }\end{array}$ & $\begin{array}{l}\text { PATIENT } \\
\text { GROUP }\end{array}$ & $\begin{array}{l}\text { PATIENT/ } \\
\text { CONTROL }\end{array}$ & $\begin{array}{c}\text { TOTAL } \\
\text { NUMBER }\end{array}$ & FEMALE & MALE & $\begin{array}{l}\text { RESEARCHED } \\
\text { PARAMETERS }\end{array}$ \\
\hline $\begin{array}{l}\text { Chaipokam et al. } \\
\text { (46) }\end{array}$ & 2017 & Thailand & $\begin{array}{c}\text { Sysmex- XE } \\
\text { Alpha }\end{array}$ & $\begin{array}{c}\text { Prospective } \\
\text { cohort }\end{array}$ & $\begin{array}{l}\text { Adult- } \\
\text { Anemia }\end{array}$ & $\begin{array}{c}\text { Control, IDA, } \\
\text { Talasemi, } \\
\text { Talasemi trait, } \\
\text { Al } \\
\end{array}$ & 267 & 190 & 77 & $\mathrm{MCV}, \mathrm{CHr}$ \\
\hline Rehu et al. (35) & 2011 & Finland & $\begin{array}{l}\text { ADVIA 120 } \\
\text { and } 2120\end{array}$ & Retrospective & $\begin{array}{l}\text { Adult- } \\
\text { Anemia }\end{array}$ & $\begin{array}{c}\text { Control, IDA, } \\
\text { ACD }\end{array}$ & 250 & 138 & 112 & $\mathrm{CHr}, \mathrm{MCV}$ \\
\hline $\begin{array}{l}\text { Brugnara et al. } \\
\text { (8) }\end{array}$ & 1999 & Amerika & $\begin{array}{c}\text { Technicon } \\
\text { H3 }\end{array}$ & & $\begin{array}{c}\text { Iron } \\
\text { Deficiency } \\
\text { Children } \\
\end{array}$ & $\begin{array}{l}\text { Control, } \\
\text { IDA, ID }\end{array}$ & 210 & 90 & 210 & $\begin{array}{c}\mathrm{CHr}, \mathrm{MCV}, \\
\text { Ferritin }\end{array}$ \\
\hline Balci et al. (47) & 2016 & Turkey & $\begin{array}{l}\text { Sysmex } \\
\text { ADVIA } \\
2120 i\end{array}$ & Case-control & $\begin{array}{l}\text { 6-12 } \\
\text { Years Old } \\
\text { Children }\end{array}$ & \begin{tabular}{|c|} 
Control, IDA, \\
$\mathrm{B}_{12}$ vitamin \\
deficiency, \\
mixed anemia \\
\end{tabular} & 112 & 52 & 60 & $\begin{array}{c}\mathrm{CHr}, \mathrm{MCV}, \\
\text { Ferritin }\end{array}$ \\
\hline Ceylan et al. (48) & 2007 & Turkey & ADVIA 120 & Case-control & $\begin{array}{l}\text { Adult- } \\
\text { Anemia }\end{array}$ & \begin{tabular}{|c|} 
Control, \\
IDA, ID, \\
Talasemi minor, \\
B $_{12}$ vitamin \\
deficiency
\end{tabular} & 131 & 91 & 40 & $\mathrm{CHr}, \mathrm{MCV}$ \\
\hline Cai et al. (49) & 2017 & China & ADVIA 120 & Case-control & Adults & $\begin{array}{c}\text { Control, IDA, } \\
\text { NIDA }\end{array}$ & 140 & 100 & 40 & $\begin{array}{c}\mathrm{CHr}, \mathrm{MCV}, \\
\text { Ferritin }\end{array}$ \\
\hline LUO et al. (50) & 2007 & China & ADVIA 120 & Case-control & \begin{tabular}{|c|} 
Pre- \\
menopausal \\
Women- \\
Anemia \\
\end{tabular} & $\begin{array}{c}\text { Control, IDA, } \\
\text { NIDA }\end{array}$ & 142 & 142 & & $\mathrm{CHr}, \mathrm{MCV}$ \\
\hline Dinh et al. (51) & 2020 & Vietnam & $\begin{array}{l}\text { Sysmex } \\
\text { ADVIA } \\
2120 i \\
\end{array}$ & Retrospective & Adult-ESRD & $\begin{array}{c}\text { Control, IDA, } \\
\text { NIDA, ESRD, } \\
\text { IDA-ESRD }\end{array}$ & 312 & 188 & 124 & $\mathrm{CHr}, \mathrm{MCV}$ \\
\hline Ageeli et al. (52) & 2013 & $\begin{array}{l}\text { Saudi } \\
\text { Arabia }\end{array}$ & $\begin{array}{l}\text { ADVIA } \\
2120 i\end{array}$ & Case-control & $\begin{array}{l}\text { Adult- } \\
\text { Anemia }\end{array}$ & $\begin{array}{l}\text { Control, IDA, } \\
\text { ACD, CRF }\end{array}$ & 320 & 170 & 150 & $\begin{array}{c}\mathrm{CHr}, \mathrm{MCV} \\
\text { Ferritin, TSAT }\end{array}$ \\
\hline $\begin{array}{l}\text { Buttarello } \\
\text { et al. (53) }\end{array}$ & 2016 & Italy & $\begin{array}{c}\text { Sysmex } \\
\text { XE-5000 }\end{array}$ & Case-control & $\begin{array}{l}\text { Adult- } \\
\text { Anemia }\end{array}$ & $\begin{array}{c}\text { Control, IDA, } \\
\text { ID, NIDA, trait } \\
\beta \text { talasemia } \\
\end{array}$ & 290 & & & $\begin{array}{l}\mathrm{CHr}, \mathrm{MCV}, \\
\text { Ferritin }\end{array}$ \\
\hline Uçar et al. (33) & 2019 & Turkey & $\begin{array}{l}\text { Sysmex } \\
\text { XN } 1000\end{array}$ & Case-control & $\begin{array}{l}\text { Adult- } \\
\text { Anemia }\end{array}$ & $\begin{array}{l}\text { Control, IDA, } \\
\text { ID, NIDA }\end{array}$ & 217 & 171 & 46 & $\begin{array}{l}\mathrm{CHr}, \mathrm{MCV} \\
\text { Ferritin, TSAT }\end{array}$ \\
\hline Toki et al. (54) & 2017 & Japan & $\begin{array}{c}\text { Sysmex XN } \\
\text { 300/XE }\end{array}$ & Case-control & $\begin{array}{l}\text { Adult- } \\
\text { Anemia }\end{array}$ & $\begin{array}{c}\text { Control, IDA, } \\
\text { ID, NIDA }\end{array}$ & 211 & 148 & 63 & $\begin{array}{c}\mathrm{CHr}, \mathrm{MCV}, \\
\text { Ferritin,TSAT }\end{array}$ \\
\hline $\begin{array}{l}\text { Vázquez-López et } \\
\text { al. (55) }\end{array}$ & 2019 & Spain & ADVIA 120 & Case-control & \begin{tabular}{|c|}
$1-16$ Years \\
Old Children \\
\end{tabular} & $\begin{array}{c}\text { Contol, IDA, } \\
\text { ID }\end{array}$ & 1239 & 620 & 619 & $\begin{array}{c}\mathrm{CHr}, \mathrm{MCV}, \\
\text { Ferritin,TSAT }\end{array}$ \\
\hline $\begin{array}{l}\text { Malczewska- } \\
\text { Lenczowska et al. } \\
(56)\end{array}$ & 2017 & Poland & ADVIA 120 & Case-control & $\begin{array}{l}\text { Sports } \\
\text { women }\end{array}$ & $\begin{array}{c}\text { Control, } \\
\text { ID stage I, } \\
\text { ID stage II }\end{array}$ & 219 & 219 & & $\begin{array}{c}\mathrm{CHr}, \mathrm{MCV}, \\
\text { Ferritin }\end{array}$ \\
\hline $\begin{array}{l}\text { Chinudomwong } \\
\text { et al. (57) }\end{array}$ & 2020 & Thailand & $\begin{array}{l}\text { Sysmex } \\
X N-10\end{array}$ & Case-control & $\begin{array}{l}\text { Adult- } \\
\text { Anemia }\end{array}$ & \begin{tabular}{|c|} 
Control, IDA, \\
IDA-inflamma- \\
tion, NIDA-AI, \\
NIDA-CKD, \\
Talasemi \\
\end{tabular} & 938 & 603 & 335 & $\begin{array}{c}\mathrm{CHr}, \mathrm{MCV}, \\
\text { Ferritin }\end{array}$ \\
\hline $\begin{array}{l}\text { Urrechaga } \\
\text { et al. (58) }\end{array}$ & 2011 & Spain & $\begin{array}{l}\text { Sysmex } \\
\text { XE } 5000\end{array}$ & Case-control & $\begin{array}{l}\text { Anemia } \\
\text { and } \\
\text { Talasemia }\end{array}$ & \begin{tabular}{|c|} 
Control, \\
talasemia trait, \\
mild IDA, \\
severe IDA \\
\end{tabular} & 473 & & & $\begin{array}{c}\mathrm{CHr}, \mathrm{MCV}, \\
\text { Ferritin, TSAT }\end{array}$ \\
\hline $\begin{array}{l}\text { Rungngu et al. } \\
\text { (59) }\end{array}$ & 2016 & Indonesia & $\begin{array}{c}\text { Sysmex } \\
\text { XE-2100 }\end{array}$ & $\begin{array}{c}\text { Cross-section- } \\
\text { al }\end{array}$ & $\begin{array}{l}\text { 6-12 } \\
\text { Years Old } \\
\text { Children- } \\
\text { Anemia }\end{array}$ & $\begin{array}{l}\text { Control, IDA, } \\
\text { NIDA }\end{array}$ & 50 & 17 & 33 & $\mathrm{CHr}$, Ferritin \\
\hline
\end{tabular}

ACD: Anemia of chronic disease, Al: Inflammation anemia, CHr: Reticulocyte hemoglobin content, CKD:Chronic kidney disease, CRF: Chronic renal failure ESRD: End-stage renal disease, ID: Iron deficiency, IDA: Iron deficiency anemia, MCV:Mean corpuscular volume, NIDA: Non-iron deficiency anemia, TM: $\beta$-Talasemia minor, TSAT: Transferrin Saturation 


\section{Publication Bias}

Two reviewers evaluated independently the risk of bias in each study using the Diagnostic Precision Study Quality Assessment Tool (QUADAS-2) recommended (18). Publication bias is demonstrated by analysis with a funnel plot.

\section{Results}

\section{Literature Search and Study Characteristics}

Based on the search strategy, 113,536 studies were evaluated among the databases specified. A total of 13,116 studies were included in the study after the records, except for studies with open access and research articles were excluded. Thirteen thou-

Table II Meta-analysis of reticulocyte hemoglobin content ( $\mathrm{CHr}$ ), Mean Corpuscular Volume (MCV), ferritin, Transferrin Saturation (TSAT), sensitivity and specificity of $\mathrm{CHr}$ to diagnose iron deficiency anemia articles.

\begin{tabular}{|c|c|c|c|c|c|c|c|c|c|c|c|c|c|c|c|c|c|c|}
\hline \multirow[b]{2}{*}{ Study } & \multirow{2}{*}{$\begin{array}{c}\text { IDA } \\
(n)\end{array}$} & \multirow{2}{*}{$\begin{array}{l}\text { IDA-CHr } \\
\text { Main } \pm \text { SD }\end{array}$} & \multirow{2}{*}{$\begin{array}{c}\text { Control } \\
(n)\end{array}$} & \multirow{2}{*}{$\begin{array}{c}\text { Control- } \\
\mathrm{CHr} \\
\text { Main } \pm \mathrm{SD}\end{array}$} & \multirow{2}{*}{$\begin{array}{c}\text { Effect } \\
\text { Size }\end{array}$} & \multirow{2}{*}{$\begin{array}{c}95 \% \\
\mathrm{Cl}\end{array}$} & \multicolumn{2}{|c|}{ MCV } & \multicolumn{2}{|c|}{ FERRITIN } & \multicolumn{2}{|c|}{ TSAT } & \multicolumn{3}{|c|}{ SENSITIVITY } & \multicolumn{3}{|c|}{ SPECIFICITY } \\
\hline & & & & & & & $\begin{array}{c}\text { EffectSi } \\
\text { ze }\end{array}$ & $\begin{array}{c}95 \% \\
\mathrm{Cl}\end{array}$ & $\begin{array}{c}\text { EffectSi } \\
\text { ze }\end{array}$ & i $\begin{array}{c}95 \% \\
\mathrm{Cl}\end{array}$ & $\begin{array}{c}\text { Effect } \\
\text { Size }\end{array}$ & $\begin{array}{c}95 \% \\
\mathrm{Cl}\end{array}$ & $\begin{array}{c}\text { Sample } \\
\text { size }\end{array}$ & $\begin{array}{c}\text { Proportion } \\
(\%)\end{array}$ & $\begin{array}{c}95 \% \\
\mathrm{Cl}\end{array}$ & $\begin{array}{c}\text { Sample } \\
\text { size }\end{array}$ & $\begin{array}{c}\text { Proportion } \\
(\%)\end{array}$ & $\begin{array}{c}95 \% \\
\mathrm{Cl}\end{array}$ \\
\hline $\begin{array}{l}\text { Chaipokam } \\
\text { et al. } 2017 \text { (46) }\end{array}$ & 53 & $21.2 \pm 5.5$ & 99 & $33.1 \pm 2.4$ & -3.137 & \begin{tabular}{|c|}
-3.62 \\
to \\
-2.65 \\
\end{tabular} & -3.30 & \begin{tabular}{|c|}
-3.80 \\
to - \\
2.80
\end{tabular} & & & & & 53 & 83.0 & $\begin{array}{c}70.2 \\
\text { to } \\
91.9 \\
\end{array}$ & 99 & 80.8 & $\begin{array}{c}71.6 \\
\text { to } \\
88.0\end{array}$ \\
\hline $\begin{array}{l}\text { Rehu et al. } \\
2011 \text { (35) }\end{array}$ & 58 & $26.8 \pm 3.8$ & 63 & $33.2 \pm 2$ & -2.12 & \begin{tabular}{|c|}
-2.57 \\
to \\
-1.67 \\
\end{tabular} & -1.16 & $\begin{array}{c}-1.54 \\
\text { to - } \\
0.77\end{array}$ & & & & & 58 & 82.7 & $\begin{array}{c}70.5 \\
\text { to } \\
91.4 \\
\end{array}$ & 63 & 90.4 & $\begin{array}{c}80.4 \\
\text { to } \\
96.4\end{array}$ \\
\hline $\begin{array}{l}\text { Brugnara } \\
\text { et al. } 1999 \text { (8) }\end{array}$ & 24 & $24.2 \pm 2.7$ & 186 & $26.8 \pm 1.8$ & -1.349 & \begin{tabular}{|c|}
-1.79 \\
to \\
-0.90 \\
\end{tabular} & -1.08 & $\begin{array}{c}-1.51 \\
\text { to }- \\
0.64\end{array}$ & -0.09 & $\begin{array}{c}-0.52 \\
\text { to } \\
0.33\end{array}$ & & & 24 & 79.1 & $\begin{array}{c}57.8 \\
\text { to } \\
92.8\end{array}$ & 186 & 74.7 & $\begin{array}{c}67.8 \\
\text { to } \\
80.8\end{array}$ \\
\hline $\begin{array}{l}\text { Balci et al. } 2016 \\
\text { (47) }\end{array}$ & 26 & $22.26 \pm 1$ & 32 & $29.9 \pm 0.7$ & -8.692 & \begin{tabular}{|c|}
-10.39 \\
to \\
-6.99 \\
\end{tabular} & -3.20 & $\begin{array}{c}-3.99 \\
\text { to }- \\
2.41\end{array}$ & -4.26 & $\begin{array}{c}-5.21 \\
\text { to } \\
-3.31\end{array}$ & & & & & & & & \\
\hline $\begin{array}{l}\text { Ceylan et al. } \\
2007 \text { (48) }\end{array}$ & 41 & $21.8 \pm 3.3$ & 34 & $28.2 \pm 1.7$ & -2.349 & \begin{tabular}{|c|}
-2.94 \\
to \\
-1.75 \\
\end{tabular} & -1.85 & $\begin{array}{c}-2.40 \\
\text { to }- \\
1.30\end{array}$ & & & & & 41 & 85.3 & $\begin{array}{c}70.8 \\
\text { to } \\
94.43\end{array}$ & 34 & 1 & $\begin{array}{c}84.6 \\
\text { to } \\
99.9\end{array}$ \\
\hline $\begin{array}{l}\text { Cai et al. } 2007 \\
\text { (49) }\end{array}$ & 56 & $23.3 \pm 4$ & 46 & $31.8 \pm 2.5$ & -2.476 & \begin{tabular}{|c|}
-2.99 \\
to \\
-1.95
\end{tabular} & -2.42 & \begin{tabular}{|c|}
-2.93 \\
to - \\
1.90
\end{tabular} & -1.91 & \begin{tabular}{|c|}
-2.38 \\
to \\
-1.44
\end{tabular} & & & 56 & 87.5 & $\begin{array}{c}75.9 \\
\text { to } \\
94.82\end{array}$ & 46 & 91.3 & $\begin{array}{c}79.2 \\
\text { to } \\
97.5\end{array}$ \\
\hline $\begin{array}{l}\text { Luo et al. } 2007 \\
(50)\end{array}$ & 30 & $23.5 \pm 3.1$ & 71 & $32 \pm 1.1$ & -4.403 & \begin{tabular}{|c|}
-5.15 \\
to \\
-3.65
\end{tabular} & $\mid-3.609$ & \begin{tabular}{|c|}
-4.27 \\
to - \\
2.94
\end{tabular} & & & & & & & & & & \\
\hline $\begin{array}{l}\text { Dinh et al. } 2020 \\
\text { (51) }\end{array}$ & 59 & $23.4 \pm 3.2$ & 145 & $31.2 \pm 1.2$ & -3.90 & \begin{tabular}{|c|}
-4.38 \\
to \\
-3.41 \\
\end{tabular} & -3.256 & \begin{tabular}{|c|}
-3.69 \\
to - \\
2.81
\end{tabular} & & & & & 59 & 98.3 & $\begin{array}{c}90.9 \\
\text { to } \\
99.9\end{array}$ & 145 & 97.9 & $\begin{array}{c}94.0 \\
\text { to } \\
99.5\end{array}$ \\
\hline $\begin{array}{l}\text { Ageeli et al. } \\
2013 \text { (52) }\end{array}$ & 100 & $22.9 \pm 2.9$ & 60 & $30.9 \pm 1.3$ & -3.27 & \begin{tabular}{|c|}
-3.76 \\
to \\
-2.79 \\
\end{tabular} & -4.093 & \begin{tabular}{|c}
-4.64 \\
to - \\
3.53
\end{tabular} & -11.85 & \begin{tabular}{|c|}
-13.2 \\
to \\
-10.5
\end{tabular} & -7.89 & \begin{tabular}{|c|}
-8.82 \\
to \\
-6.96
\end{tabular} & & & & & & \\
\hline $\begin{array}{l}\text { Buttarello } \\
\text { et al. } 2016 \text { (53) }\end{array}$ & 58 & $24.4 \pm 4.8$ & 164 & $33 \pm 1.2$ & -3.23 & \begin{tabular}{|c|}
-3.66 \\
to \\
-2.80
\end{tabular} & -2.141 & \begin{tabular}{|c|}
-2.50 \\
to - \\
1.78
\end{tabular} & -1.31 & \begin{tabular}{|c|}
-1.63 \\
to \\
-0.99
\end{tabular} & & & 58 & 91.3 & $\begin{array}{c}81.0 \\
\text { to } \\
97.1\end{array}$ & 164 & 94.5 & $\begin{array}{c}89.8 \\
\text { to } \\
97.4\end{array}$ \\
\hline $\begin{array}{l}\text { Uçar et al. } 2019 \\
\text { (33) }\end{array}$ & 52 & $21 \pm 4.1$ & 54 & $36.6 \pm 7$ & -2.69 & \begin{tabular}{|c|}
-3.22 \\
to \\
-2.15 \\
\end{tabular} & -2.996 & $\begin{array}{c}-3.55 \\
\text { to }- \\
2.43\end{array}$ & -1.10 & \begin{tabular}{|c|}
-1.51 \\
to \\
-0.69
\end{tabular} & -2.83 & $\begin{array}{c}-3.37 \\
\text { to } \\
-2.29\end{array}$ & 52 & 90.3 & \begin{tabular}{|c|}
78.9 \\
to \\
96.8 \\
\end{tabular} & 54 & 100 & $\begin{array}{c}93.3 \\
\text { to } \\
100.0\end{array}$ \\
\hline $\begin{array}{l}\text { Toki et al. } 2017 \\
\text { (54) }\end{array}$ & 72 & $23.4 \pm 4.9$ & 67 & $33.8 \pm 2.5$ & -2.631 & \begin{tabular}{|c|}
-3.08 \\
to \\
-2.17
\end{tabular} & -2.008 & $\begin{array}{c}-2.41 \\
\text { to - } \\
1.59\end{array}$ & -0.56 & \begin{tabular}{|c|}
-0.90 \\
to \\
-0.22
\end{tabular} & -2.38 & \begin{tabular}{|c|}
-2.82 \\
to \\
-1.94
\end{tabular} & & & & & & \\
\hline $\begin{array}{l}\text { Vázquez-L } \\
\text { et al. } 2019 \text { (55) }\end{array}$ & 13 & $24.5 \pm 30$ & 1153 & $31.1 \pm 1.7$ & -1.885 & \begin{tabular}{|c|}
-2.43 \\
to \\
-1.33 \\
\end{tabular} & -2.951 & \begin{tabular}{|c|}
-3.51 \\
to - \\
2.39
\end{tabular} & -1.64 & \begin{tabular}{|c|}
-2.19 \\
to \\
-1.09 \\
\end{tabular} & -1.51 & $\begin{array}{c}-2.06 \\
\text { to } \\
-0.96\end{array}$ & & & & & & \\
\hline $\begin{array}{l}\text { Malczewska-L } \\
\text { et al. } 2017 \text { (56) }\end{array}$ & 33 & $29.3 \pm 1.8$ & 87 & $31.4 \pm 1.0$ & -1.567 & \begin{tabular}{|c|}
-2.02 \\
to \\
-1.12
\end{tabular} & -0.457 & $\begin{array}{c}-0.86 \\
\text { to } \\
-0.05\end{array}$ & -2.44 & \begin{tabular}{|c|}
-2.94 \\
to \\
-1.93
\end{tabular} & & & & & & & & \\
\hline $\begin{array}{l}\text { Chinudomwong } \\
\text { et al. } 2020 \text { (57) }\end{array}$ & 133 & $20.6 \pm 9$ & 155 & $33 \pm 1.4$ & -1.993 & \begin{tabular}{|c|}
-2.27 \\
to \\
-1.71 \\
\end{tabular} & $\mid-1.909$ & $\begin{array}{c}-2.18 \\
\text { to } \\
-1.63\end{array}$ & -1.40 & $\begin{array}{c}-1.66 \\
\text { to } \\
-1.14\end{array}$ & & & 133 & 73.6 & $\begin{array}{c}65.3 \\
\text { to } \\
80.9 \\
\end{array}$ & 155 & 96.7 & $\begin{array}{c}92.6 \\
\text { to } \\
98.9\end{array}$ \\
\hline $\begin{array}{l}\text { Urrechaga } \\
\text { et al. } 2011 \text { (58) }\end{array}$ & 126 & $22.3 \pm 3.7$ & 90 & $33.7 \pm 1.4$ & -3.827 & \begin{tabular}{|c|}
-4.28 \\
to \\
-3.37 \\
\end{tabular} & -3.235 & $\begin{array}{c}-3.64 \\
\text { to } \\
-2.82\end{array}$ & -2.44 & $\begin{array}{c}-2.80 \\
\text { to } \\
-2.08 \\
\end{array}$ & -4.39 & $\begin{array}{c}-4.89 \\
\text { to } \\
-3.89\end{array}$ & & & & & & \\
\hline $\begin{array}{l}\text { Rungngu } \\
\text { et al. } 2016 \text { (59) }\end{array}$ & 16 & $25.8 \pm 4.8$ & 34 & $29.8 \pm 1.3$ & -1.338 & \begin{tabular}{|c|}
-1.99 \\
to \\
-0.68
\end{tabular} & & & -1.59 & $\begin{array}{c}-2.27 \\
\text { to } \\
-0.91\end{array}$ & & & 16 & 43.7 & $\begin{array}{c}19.7 \\
\text { to } \\
70.1\end{array}$ & 34 & 85.2 & $\begin{array}{c}68.9 \\
\text { to } \\
95.0\end{array}$ \\
\hline $\begin{array}{l}\text { Total (random } \\
\text { effects) }\end{array}$ & 950 & & 2540 & & -2.846 & $\begin{array}{c}-3.31 \\
\text { to } \\
-2.38\end{array}$ & -2.463 & $\begin{array}{c}-2.95 \\
\text { to } \\
-1.97\end{array}$ & -2.38 & $\begin{array}{c}-3.11 \\
\text { to } \\
-1.63\end{array}$ & -3.77 & $\begin{array}{l}-5.38 \\
\text { to } \\
-2.15\end{array}$ & 550 & 83.5 & $\begin{array}{c}76.1 \\
\text { to } \\
89.9\end{array}$ & 980 & 91.8 & $\begin{array}{l}85.6 \\
\text { to } \\
96.4\end{array}$ \\
\hline
\end{tabular}

$\mathrm{Cl}=$ Confidenceinterval, $\mathrm{n}=$ Sample size, $\mathrm{SD}=$ Standard Deviation 


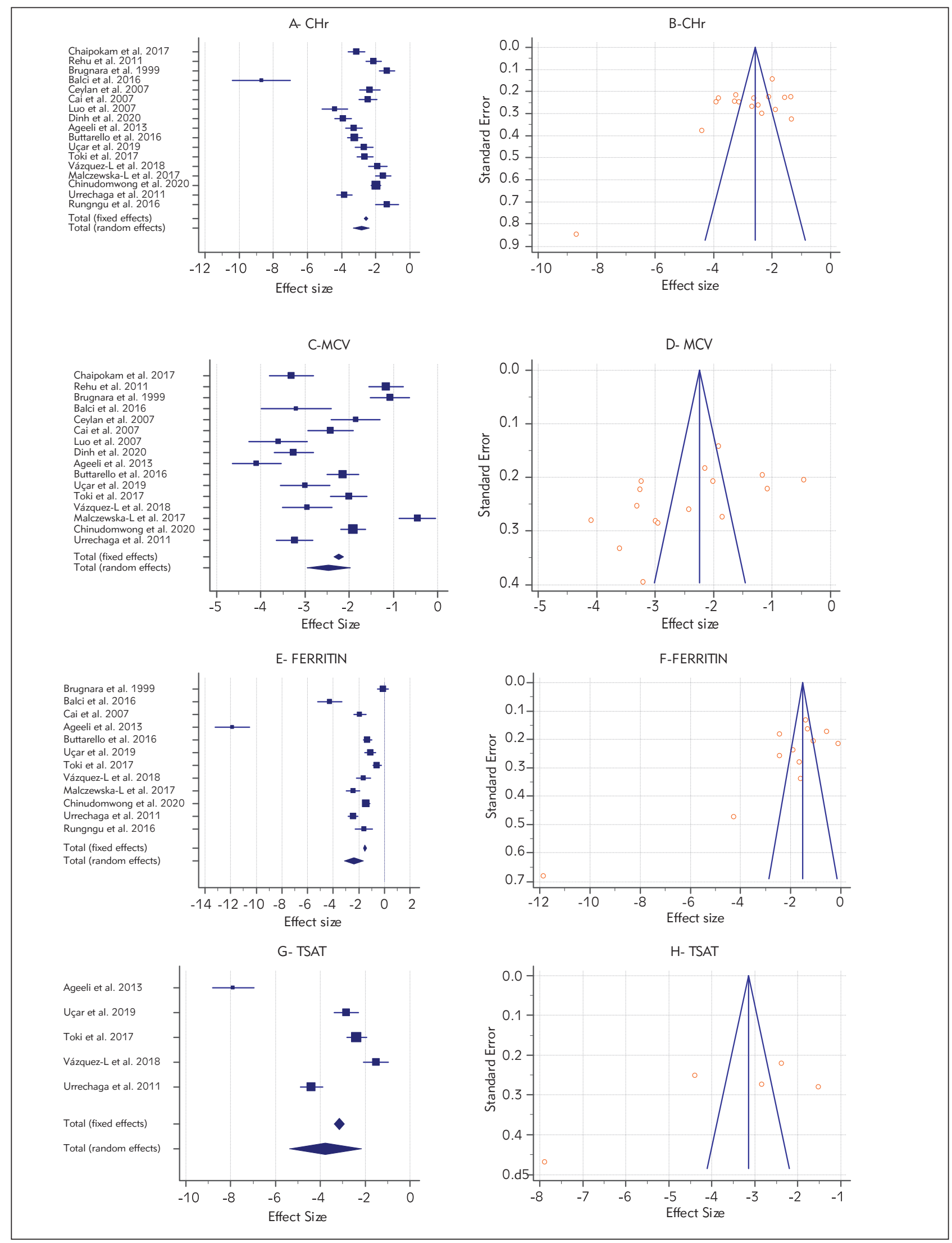

Figure 2A Forest plot of reticulocyte hemoglobin content $(\mathrm{CHr})$, B Funnel plot of reticulocyte hemoglobin content, $\mathbf{C}$ Forest plot of mean corpuscular volume (MCV), D Funnel plot of mean corpuscular volume, E Forest plot of ferritin, F Funnel plot of ferritin, G Forest plot of transferrin saturation (TSAT), H Funnel plot of transferrin saturation. 
sand twenty-two articles not suitable for the title and abstracts and published in languages other than English were excluded. From the remaining 94 pieces, 17 of them were included in the meta-analysis, excluding those with repeating records, animal studies, those that did not include a control group, and those related to chronic diseases other than iron deficiency. The flowchart and detailed literature search steps are shown in Figure 1.

\section{Characteristics and Quality Studies}

The articles on $\mathrm{CHr}$, a biomarker used in patients with IDA, were included in our study, all in English until December 29, 2020. From the 17 articles, 2 of them were written in Thailand, 3 in Turkey, 2 in Spain, 1 in Finland, 1 in the United States, 2 in China, and one in Indonesia, Vietnam, Saudi Arabia, Italy, Poland, and Japan. The study contains 951 individuals with IDA and 3491 people, including 2540 control group members. The features of the included studies are shown in Figure 1.

\section{Reticulocyte Hemoglobin Content}

As a result of a detailed literature review, 17 articles including IDA data of $\mathrm{CHr}$ were included. In the meta-analysis performed with the low bias risk random-effects model in the included articles, the $\mathrm{I}^{2}$ value of $\mathrm{CHr}$ was $93.52 \%$, so intergroup heterogeneity was achieved $(P<0.0001)$, and random effect size value 2.84 (95\% Cl 2.36 to 3.31$)$ is shown in Table II. Publication bias is evaluated with a forest plot in Figure $2 A$ and funnel plot in Figure $2 B$.

\section{Mean Corpuscular Volume}

After the literature review, 16 articles with MCV data were included. In the meta-analysis performed with the low bias risk random-effects model in the included articles, the MCV I ${ }^{2}$ value was $94.71 \%$, so intergroup heterogeneity was achieved $(P<0.0001)$, and random effect size value $2.46(95 \% \mathrm{Cl} 1.97$ to 2.95 ) is shown in Table II. Publication bias is evaluated with a forest plot Figure $2 \mathrm{C}$ and funnel plot in Figure 2D.

\section{Ferritin}

12 articles with ferritin data were included. In the meta-analysis performed with the low bias risk random-effects model in the included articles, the ferritin $1^{2}$ value was $97.15 \%$, so intergroup heterogeneity was achieved $(P<0.0001)$, and random effect size value 2.37 (95\% Cl 1.63 to 3.11 ) is shown in Table II. Publication bias is evaluated with a forest plot Figure $2 E$ and funnel plot in Figure $2 F$.

\section{Transferrin Saturation}

5 articles with TSAT data were included. In the meta-analysis performed with the low bias risk model of random effects in the included articles, the TSAT I ${ }^{2}$ value was $97.70 \%$. Therefore, intergroup heterogeneity was achieved $(P<0.0001)$, and random effect size value $3.76(95 \% \mathrm{Cl} 2.14$ to 5.38$)$ is shown in Table II. Publication bias is evaluated with a forest plot Figure $2 \mathrm{G}$ and funnel plot in Figure $2 \mathrm{H}$.

\section{Discussion}

Anemia is a global health problem that is quite common worldwide and affects $43 \%$ of children under the age of five, $38 \%$ of pregnant women, and $29 \%$ of non-pregnant women (19). IDA brings along many complications such as growth retardation, neurocognitive deficiencies, impaired immune system, increased risk of premature, and impaired learning ability (20-24). It is therefore important to ensure accurate and timely diagnosis of the disease by preventing such adverse effects (25-27). Although there is no single and best test for determining iron deficiency, bone marrow aspiration, which is accepted as the gold standard, and the method of staining bone marrow macrophages and erythroid precursors with Prussian blue is used. However, the method is not suitable for routine use because it is expensive, subjective, and invasive $(26,28)$.

There are many biochemical parameters used in the diagnosis of IDA. However, as these parameters are affected by certain conditions, it is not easy to evaluate them. Serum ferritin concentration, serum iron level, TSAT, and total iron-binding capacity (TIBC) are the most widely used biochemical tests. Although serum ferritin level reveals the iron concentration accumulated in the body, factors such as acute and chronic inflation, malignancy, liver diseases, and excessive alcohol use increase independent of iron (29). Serum iron level decreases with infection, inflammation, and malignancy but increases with liver disease. Since the TSAT level is calculated on iron and TIBC, it is affected by changes in these values and does not always give an accurate result (29-32).

In recent years, $\mathrm{CHr}$ has become one of the parameters used to determine IDA $(33,34)$. reticulocytes, as the first erythrocytes produced in the bone marrow, transform into mature red blood cells a day or two after entering the bloodstream. Reticulocyte parameters have become one of the parameters used to reflect the iron status in a short time due to their shorter lifespan compared to erythrocytes and ability to provide information about bone marrow erythrocyte production $(12,35)$. CHr has a higher specificity and a lower coefficient of variation since it is not affected by inflammation like some parameters used in the diagnosis of IDA (36). Reticulocyte hemoglobin content data can be obtained from a few millimeters 
SENSITIVITY

Chaipokam et al. 2017

Rehu et al. 2011

Brugnara et al. 1999

Ceylan et al. 2007

Cai et al. 2007

Dinh et al. 2020

Buttarello et al. 2016

Uçar et al. 2019

Chinudomwong et al. 2020

Rungngu et al. 2016

Total (fixed effects)

Total (random effects)

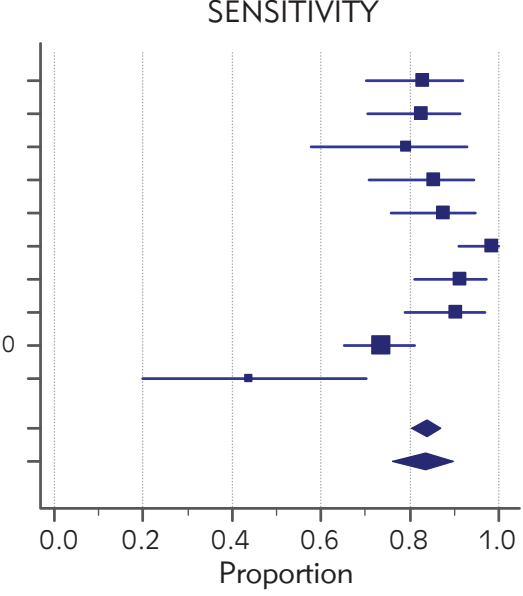

A

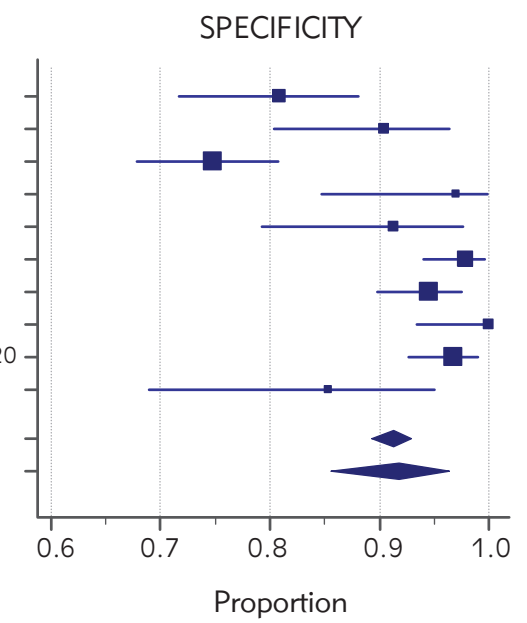

C

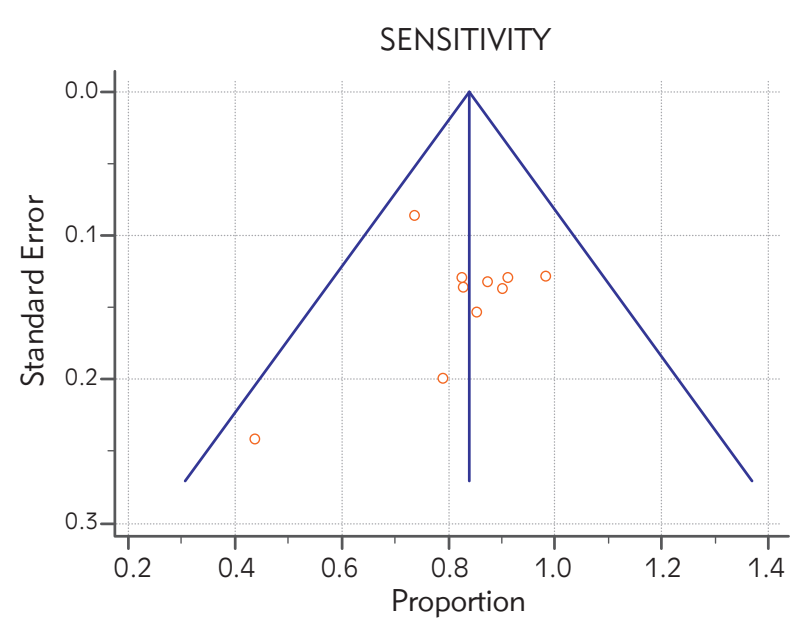

B



D
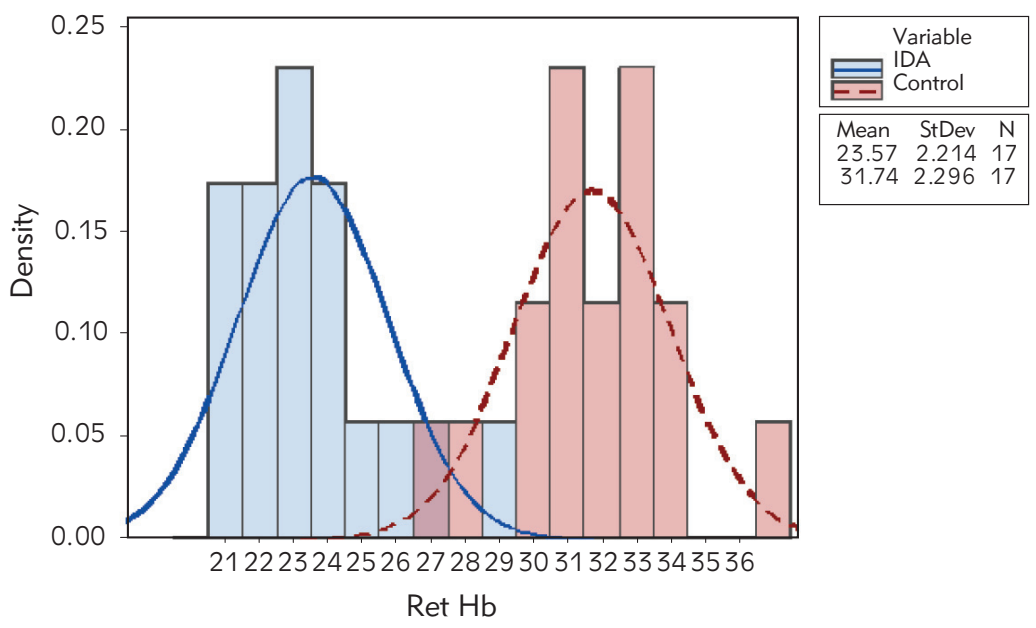

Figure 3A Forest plot meta-analysis of the sensitivity of reticulocyte hemoglobin content to diagnose iron-deficiency anemia articles, B Funnel plot meta-analysis of the sensitivity of reticulocyte hemoglobin content to diagnose iron-deficiency anemia articles, C Forest plot meta-analysis of the specificity of reticulocyte hemoglobin content to diagnose iron-deficiency anemia articles, D Funnel plot meta-analysis of the specificity of reticulocyte hemoglobin content to diagnose iron-deficiency anemia articles, the reticulocyte hemoglobin content mean cut-off value. 


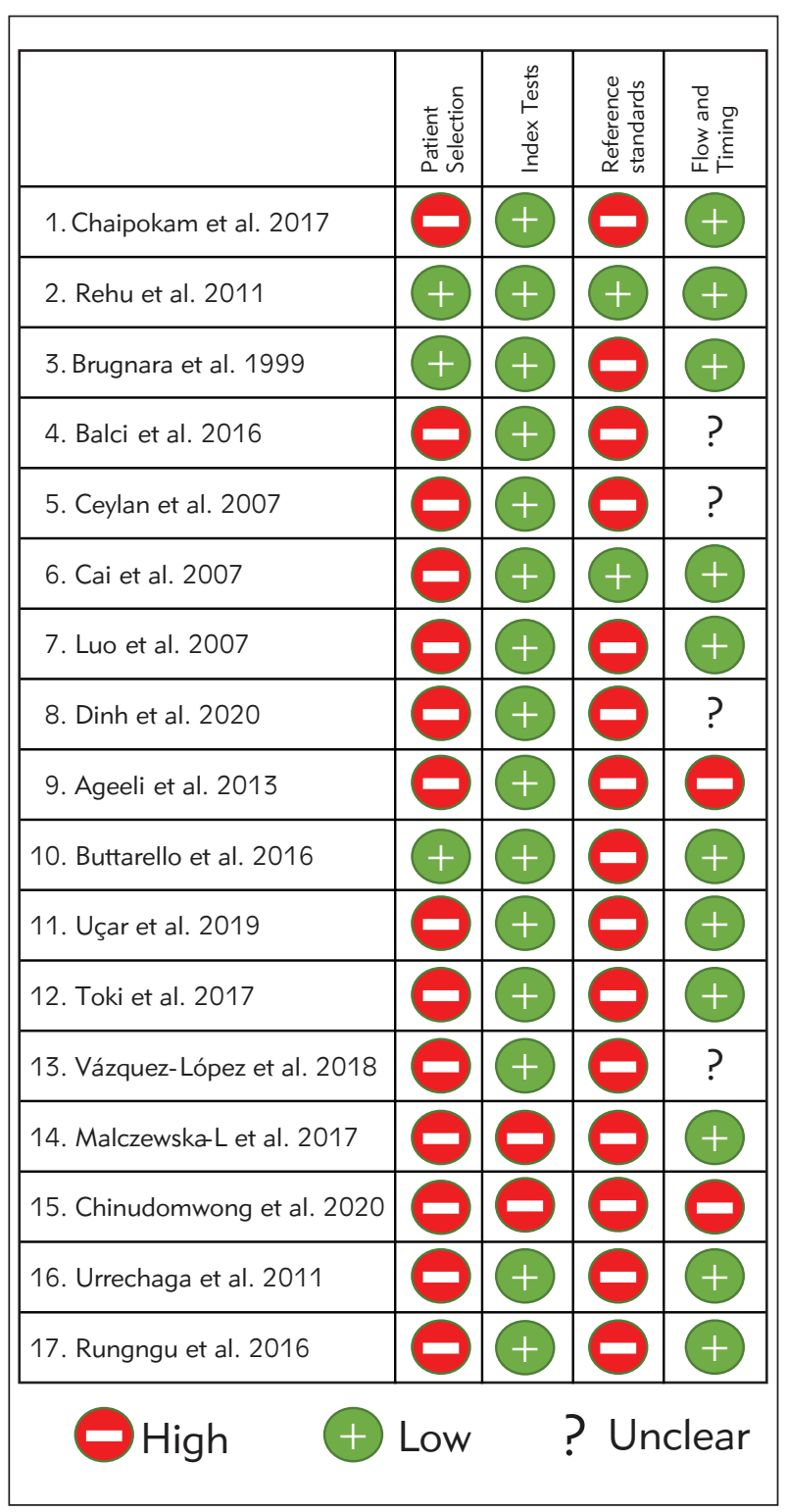

Figure 4 Methodological quality of the included studies (individual assessment).

of peripheral blood compared to bone marrow biopsy and is also advantageous because it is relatively inexpensive, convenient, and less invasive (37).

In this random-effect meta-analysis, studies on the efficiency of $\mathrm{CHr}$ in the diagnosis of IDA are summarized. For this meta-analysis, a total of 13,116 articles were examined, and 17 studies were included in the study according to the inclusion and exclusion criteria. The number of articles including parameters such as MCV, ferritin, and TSAT, which were used frequently in the past in the diagnosis of iron deficiency, varies (16 articles for MCV values, 12 articles for Ferritin values, and 5 articles for TSAT values were examined). The most effective examination is thought to be obtained by including each study that meets the criteria given in Figure 1 for meta-analysis. The litera- ture review exhibited the fact that this study is the first meta-analysis of $\mathrm{CHr}$ to determine the diagnosis of IDA.

A total of 3491 individuals, of which 2540 people were in the control group, and 951 people were with IDA, were included in this study. The inclusion criteria for the current study were determined as $\mathrm{CHr}$, which is one of the important parameters in the diagnosis of IDA and containing at least one of the parameters of MCV, ferritin, or TSAT.

Ferritin is an indicator of the total amount of iron stored in the body. When the serum ferritin level shows values less than $15 \mathrm{mg} / \mathrm{L}$, it is highly suggestive for the diagnosis of IDA. At values below 30 $\mathrm{mg} / \mathrm{L}$, the sensitivity is $92 \%$, and the specificity is $98 \%$ $(38,39)$. Again, a ferritin level below $45 \mathrm{mg} / \mathrm{L}$ and the sensitivity of $85 \%$ and specificity of $92 \%$ are particular to IDA $(40,41)$.

A low MCV value alone is not sufficient for a diagnosis of IDA. Unless the MCV volume is greater than $95 \mu \mathrm{m}$ ] ( $95 \mathrm{fL}$ ), it should not be considered in IDA because this threshold has a sensitivity of $97.6 \%$ $(40,42)$.

TSAT is low in IDA, typically less than 10\%, and in this case, the sensitivity is $48 \%$, and the specificity is $88 \%(43,44)$.

In the meta-analysis performed with the low bias risk random-effects model in the included articles, the sensitivity $\mathrm{CHr} \mathrm{I}^{2}$ value was $78.78 \%(95 \% \mathrm{Cl} 61.43$ to 88.32), so intergroup heterogeneity was achieved $(\mathrm{P}<0.0001)$ (Figure 3A-B). The sensitivity of $\mathrm{CHr}$ to diagnose IDA was found as $83.5 \%(95 \% \mathrm{Cl} 76.1$ to 89.8), and they are shown in Table II.

In the meta-analysis performed with the low bias risk random-effects model in the included articles, the specificity $\mathrm{CHr} \mathrm{I}^{2}$ value was $89.53 \%(95 \% \mathrm{Cl} 82.88$ to 93.60), so intergroup heterogeneity was achieved $(\mathrm{P}<0.0001)$ (Figure 3C-D). The sensitivity of $\mathrm{CHr}$ to diagnose IDA was found as $91.8 \%(95 \% \mathrm{Cl} 85.5$ to 96.4), and they are shown in Table II.

In conclusion, the meta-analysis study showed that $\mathrm{CHr}$ is a better marker than other more commonly used parameters in IDA. Many previous studies also support this conclusion. Also, this meta-analysis we conducted is important for being the first meta-analysis study regarding $\mathrm{CHr}$ and IDA.

The literature review revealed that different cutoff results related to $\mathrm{CHr}$ were obtained, and these values vary between $28-29 \mathrm{pg}$. The $\mathrm{CHr}$ mean cutoff value obtained in our study is 28.2, and this is shown in the histogram in Figure $3 E$.

There are important heterogeneity and bias problems in the studies. Patient selection and lack of reference methods are particularly important. There are important criteria differences for index tests. Also, 
it is important to have significant group differences (pediatric patients, renal failure, etc.) in studies. It should be noted that there will be a significant change in methodologies over time (Figure 4).

All parameters of IDA, which are included in the research, have heterogeneity. $\mathrm{CHr}$ is a more effective marker in determining IDA compared to the routinely used MCV and ferritin levels. The effect size value of TSAT, one of the parameters examined, is above 0.80 , and its selectivity is higher than $\mathrm{CHr}$.

The study also has some limitations. These are as follows: like other meta-analysis studies in the literature, methodological differences are arising from combining studies conducted with different methods, and this may lead to bias. Since studies in which the diagnosis of IDA of $\mathrm{CHr}$ was evaluated in the metaanalysis were included, many parameters used routinely were excluded. IDA, erythropoiesis status, and chronic anemias were excluded from the study. In the study, no distinction was made according to gender and age.

\section{Conclusion}

This study is the first meta-analysis to evaluate the efficiency of $\mathrm{CHr}$ in the diagnosis of IDA.

\section{References}

1. Baker RD, Greer FR. Diagnosis and Prevention of Iron Deficiency and Iron-Deficiency Anemia in Infants and Young Children (0-3 Years of Age). Pediatrics [Internet]. 2010 Nov 1; 126(5): 1040-50. Available from: http://pediatrics.aappublications.org/cgi/doi/10.1542/ peds.2010-2576

2. Aslan Y, Erduran E, Mocan H, Gedik Y, Okten A, Soylu H, $O$ Değer. Absorption of iron from grape-molasses and ferrous sulfate: a comparative study in normal subjects and subjects with iron deficiency anemia. Turk J Pediatr 1997; 39: 465-71.

3. Guralnik JM, Eisenstaedt RS, Ferrucci L, Klein HG, Woodman RC. Prevalence of anemia in persons 65 years and older in the United States: evidence for a high rate of unexplained anemia. Blood [Internet]. 2004 Oct 15; 104(8): 2263-8. Available from: https://ashpublications.org/blood/article/104/8/2263/19067/Prevalence -of-anemia-in-persons-65-years-and-older

4. Joosten E. Iron deficiency anemia in older adults: A review. Geriatr Gerontol Int [Internet]. 2018 Mar; 18(3): 373-9. Available from: http://doi.wiley.com/10.1111/ ggi.13194

5. Agha F, Sadaruddin A, Khan RA, Ghafoor A. Iron deficiency in adolescents. J Pak Med Assoc [Internet]. 1992 Jan; 42(1): 3-5. Available from: http://www.ncbi.nlm. nih.gov/pubmed/1545482
According to our results, $\mathrm{CHr}$ should be used additionally with the parameters used in the diagnosis of IDA. 'The results of our study reveal the findings that $\mathrm{CHr}$ is a better biomarker than MCV and ferritin used in determining IDA, and its efficacy is lower than TSAT. It is very important to routinely use it for the pre-diagnosis of IDA, which is very important for public health.' CHr alone provides important information about the current bioavailability of iron, but its use with other parameters removes uncertainty about the diagnosis and treatment of IDA. CHr is a very important parameter that can be used to evaluate a very common disease in the clinic, such as IDA' The heterogeneity index of the study results is quite high. Therefore, comprehensive studies with more homogeneous groups are needed to elucidate the relationship between IDA and $\mathrm{CHr}$.

Acknowledgements. Not applicable.

\section{Conflict of interest statement}

All the authors declare that they have no conflict of interest in this work.

6. McMahon LP. Iron deficiency in pregnancy. Obstet Med [Internet]. 2010 Mar 4; 3(1): 17-24. Available from: http://journals.sagepub.com/doi/10.1258/om.2010.1 00004

7. Kim BSM. Diagnosis of gastrointestinal bleeding: A practical guide for clinicians. World J Gastrointest Pathophysiol [Internet]. 2014; 5(4): 467. Available from: http://www.wjgnet.com/2150-5330/full/v5/ i4/467.htm

8. Brugnara C. Reticulocyte Hemoglobin Content to Diagnose Iron Deficiency in Children. JAMA [Internet]. 1999 Jun 16; 281(23): 2225. Available from: http://jama.jamanetwork.com/article. aspx?doi=10.100 1/jama.281.23.2225

9. Powers JM, Buchanan GR, Adix L, Zhang S, Gao A, McCavit TL. Effect of Low-Dose Ferrous Sulfate vs Iron Polysaccharide Complex on Hemoglobin Concentration in Young Children With Nutritional Iron-Deficiency Anemia. JAMA [Internet]. 2017 Jun 13; 317(22): 2297. Available from: http://jama.jamanetwork.com/article. aspx?doi=10.1001/jama.2017.6846

10. Ogawa C, Tsuchiya K, Maeda K. Reticulocyte hemoglobin content. Clin Chim Acta [Internet]. 2020 May; 504: 138-45. Available from: https://linkinghub.elsevier. com/retrieve/pii/S0009898120300528

11. Mast AE, Blinder MA, Dietzen DJ. Reticulocyte hemoglobin content. Am J Hematol [Internet]. 2008 Apr; 
83(4): 307-10. Available from: http://doi.wiley.com/ 10.1002/ajh.21090

12. Ullrich C. Screening Healthy Infants for Iron Deficiency Using Reticulocyte Hemoglobin Content. JAMA [Internet]. 2005 Aug 24; 294(8): 924. Available from: http://jama.jamanetwork.com/article.aspx?doi=10.10 01/jama.294.8.924

13. Buttarello $M$, Pajola $R$, Novello E, Rebeschini $M$, Cantaro S, Oliosi F, et al. Diagnosis of Iron Deficiency in Patients Undergoing Hemodialysis. Am J Clin Pathol [Internet]. 2010 Jun 1; 133(6): 949-54. Available from: https://academic.oup.com/ajcp/article-lookup/ doi/10.1309/AJCPQAXOJFHFSOOA

14. Chuang C-L. Early prediction of response to intravenous iron supplementation by reticulocyte haemoglobin content and high-fluorescence reticulocyte count in haemodialysis patients. Nephrol Dial Transplant [Internet]. 2003 Feb 1; 18(2): 370-7. Available from: https://academic.oup.com/ndt/article-lookup/doi/ 10.1093/ndt/18.2.370

15. Gaweda AE. Markers of iron status in chronic kidney disease. Hemodial Int [Internet]. 2017 Apr; 21: S21-7. Available from: http://doi.wiley.com/10.1111/hdi. 12556

16. Mittman N, Sreedhara R, Mushnick R, Chattopadhyay J, Zelmanovic D, Vaseghi $M$, et al. Reticulocyte hemoglobin content predicts functional iron deficiency in hemodialysis patients receiving $\mathrm{rHuEPO}$. Am J Kidney Dis [Internet]. 1997 Dec; 30(6): 912-22. Available from: https://linkinghub.elsevier.com/retrieve/pii/ S0272638697901049

17. Majoni SW, Lawton PD, Rathnayake G, Barzi F, Hughes JT, Cass A. Narrative Review of Hyperferritinemia, Iron Deficiency, and the Challenges of Managing Anemia in Aboriginal and Torres Strait Islander Australians With CKD. Kidney Int Reports [Internet]. 2020 Nov; Available from: https://linkinghub.elsevier.com/ retrieve/pii/S246802492031706X

18. Whiting PF. QUADAS-2: A Revised Tool for the Quality Assessment of Diagnostic Accuracy Studies. Ann Intern Med [Internet]. 2011 Oct 18; 155(8): 529. Available from: http://annals.org/article.aspx?doi=10.7326/ 0003-4819-155-8-201110180-00009

19. Hastka J, Lasserre JJ, Schwarzbeck A, Hehlmann R. Central role of zinc protoporphyrin in staging iron deficiency. Clin Chem [Internet]. 1994 May 1; 40(5): 76873. Available from: https://academic.oup.com/ clinchem/article/40/5/768/5647571

20. Pasricha S-R, Drakesmith H. Iron Deficiency Anemia. Hematol Oncol Clin North Am [Internet]. 2016 Apr; 30(2): 309-25. Available from: https://linkinghub. elsevier.com/retrieve/pii/S0889858815001902

21. Kiudelienè R, Griniūtè R, Labanauskas L. Prognostic value of reticulocyte hemoglobin content to diagnose iron deficiency in 6-24-month-old children. Medicina (B Aires) [Internet]. 2008 Sep 15; 44(9): 673. Available from: http://www.mdpi.com/1010-660X/44/9/673

22. Hempel E V, Bollard ER. The Evidence-Based Evaluation of Iron Deficiency Anemia. Med Clin North Am [Internet]. 2016 Sep; 100(5): 1065-75. Available from: https://linkinghub.elsevier.com/retrieve/pii/ S0025712516372790

23. Turgeon $O^{\prime}$ Brien $H$, Blanchet $R$, Gagné $D$, Lauzière J, Vézina C. Using Soluble Transferrin Receptor and Taking Inflammation into Account When Defining Serum Ferritin Cutoffs Improved the Diagnosis of Iron Deficiency in a Group of Canadian Preschool Inuit Children from Nunavik. Anemia [Internet]. 2016; 2016: 1-10. Available from: http://www.hindawi.com/ journals/anemia/2016/6430214/

24. Kassebaum NJ. The Global Burden of Anemia. Hematol Oncol Clin North Am [Internet]. 2016 Apr; 30(2): 247-308. Available from: https://linkinghub. elsevier.com/retrieve/pii/S0889858815001896

25. Ennis KM, Dahl L V, Rao RB, Georgieff MK. Reticulocyte hemoglobin content as an early predictive biomarker of brain iron deficiency. Pediatr Res [Internet]. 2018 Nov 13; 84(5): 765-9. Available from: http://www.nature.com/articles/s41390-018-0178-6

26. Lopez A, Cacoub P, Macdougall IC, Peyrin-Biroulet L. Iron deficiency anaemia. Lancet [Internet]. 2016 Feb; 387(10021): 907-16. Available from: https://linkinghub.elsevier.com/retrieve/pii/S0140673615608650

27. Mehta S, Goyal LK, Kaushik D, Gulati S, Sharma N, Harshvardhan L, et al. Reticulocyte Hemoglobin vis-avis Serum Ferritin as a Marker of Bone Marrow Iron Store in Iron Deficiency Anemia. J Assoc Physicians India [Internet]. 2016; 64(11): 38-42. Available from: http://www.ncbi.nlm.nih.gov/pubmed/27805332

28. Braga F, Infusino I, Dolci A, Panteghini M. Soluble transferrin receptor in complicated anemia. Clin Chim Acta [Internet]. 2014 Apr; 431: 143-7. Available from: https://linkinghub.elsevier.com/retrieve/pii/S0009898 114000709

29. Jimenez K, Kulnigg-Dabsch S, Gasche C. Management of Iron Deficiency Anemia. Gastroenterol Hepatol (N Y) [Internet]. 2015 Apr; 11(4): 241-50. Available from: http://www.ncbi.nlm.nih.gov/pubmed/27099596

30. Fishbane S, Galgano C, Langley RC, Canfield W, Maesaka JK. Reticulocyte hemoglobin content in the evaluation of iron status of hemodialysis patients. Kidney Int [Internet]. 1997 Jul; 52(1): 217-22. Available from: https://linkinghub.elsevier.com/retrieve/pii/S0085253 815601636

31. Karagülle M, Gündüz E, Mutlu FŞ, Akay MO. Clinical Significance of Reticulocyte Hemoglobin Content in the Diagnosis of Iron Deficiency Anemia. Turkish J Hematol [Internet]. 2013 Jun 5; 30(2): 153-6. Available from: http://www.journalagent.com/z4/download fulltext.as p?pdir $=$ tjh\&plng $=$ eng\&un $=\mathrm{TJH}-59320$

32. Duman E, Kulaksızoglu S, Çifçi E, Ozulku M. Is There a Real Correlation Between Red Cell Distribution Width and Peripheral Arterial Disease? J Med Biochem [Internet]. 2017 Aug 28; 36(4): 309-13. Available from: https://scindeks.ceon.rs/article.aspx?artid= 1452-82581704309D

33. Uçar MA, Falay M, Dağdas S, Ceran F, Urlu SM, Özet $\mathrm{G}$. The importance of RET-He in the diagnosis of iron deficiency and iron deficiency anemia and the evaluation of response to oral iron therapy. J Med Biochem 
[Internet]. 2019 Jan 22; 38(4): 496-502. Available from: https://scindeks.ceon.rs/article.aspx?artid= 1452-82581904496A

34. Bahrainwala J, Berns JS. Diagnosis of Iron-Deficiency Anemia in Chronic Kidney Disease. Semin Nephrol [Internet]. 2016 Mar; 36(2): 94-8. Available from: https://linkinghub.elsevier.com/retrieve/pii/S0270929 516000139

35. Rehu M, Ahonen S, Punnonen K. The diagnostic accuracy of the percentage of hypochromic red blood cells (\%HYPOm) and cellular hemoglobin in reticulocytes $(\mathrm{CHr})$ in differentiating iron deficiency anemia and anemia of chronic diseases. Clin Chim Acta [Internet]. 2011 Sep; 412(19-20): 1809-13. Available from: https://linkinghub.elsevier.com/retrieve/pii/S0009898 111003160

36. Kotisaari S, Romppanen J, Penttilä I, Punnonen K. The Advia 120 red blood cell and reticulocyte indices are useful in diagnosis of iron-deficiency anemia. Eur J Haematol [Internet]. 2002 Mar; 68(3): 150-6. Available from: http://doi.wiley.com/10.1034/j.16000609.2002.01532.x

37. Fishbane S, Shapiro W, Dutka P, Valenzuela OF, Faubert J. A randomized trial of iron deficiency testing strategies in hemodialysis patients11See Editorial by Besarab, p. 2412. Kidney Int [Internet]. 2001 Dec; 60(6): 240611. Available from: https://linkinghub.elsevier.com/ retrieve/pii/S0085253815481360

38. Ermens AAM, Hoffmann JJML, Krockenberger M, Van Wijk EM. New erythrocyte and reticulocyte parameters on CELL-DYN Sapphire: analytical and preanalytical aspects. Int J Lab Hematol [Internet]. 2012 Jun; 34(3): 274-82. Available from: http://doi.wiley.com/ 10.1111/j.1751-553X.2011.01391.x

39. Camaschella C. Iron-Deficiency Anemia. Longo DL, editor. N Engl J Med [Internet]. 2015 May 7; 372(19): 1832-43. Available from: http://www.nejm.org/doi/ 10.1056/NEJMra1401038

40. Mast AE, Blinder MA, Gronowski AM, Chumley C, Scott MG. Clinical utility of the soluble transferrin receptor and comparison with serum ferritin in several populations. Clin Chem [Internet]. 1998 Jan; 44(1): 45-51. Available from: http://www.ncbi.nlm.nih.gov/pubmed/ 9550557

41. loannou GN, Spector J, Scott K, Rockey DC. Prospective evaluation of a clinical guideline for the diagnosis and management of iron deficiency anemia. Am J Med [Internet]. 2002 Sep; 113(4): 281-7. Available from: https://linkinghub.elsevier.com/retrieve/pii/ S0002934302012263

42. Guyatt GH, Patterson C, Ali M, Levine M, Turpie I, Meyer $\mathrm{R}$, et al. Diagnosis of iron-deficiency anemia in the elderly. Am J Med [Internet]. 1990 Mar; 88(3): 205-9. Available from: https://linkinghub.elsevier. com/retrieve/pii/0002934390901432

43. Bermejo F, García-López S. A guide to diagnosis of iron deficiency and iron deficiency anemia in digestive diseases. World J Gastroenterol [Internet]. 2009; 15(37): 4638. Available from: http://www.wjgnet.com/10079327/full/v15/i37/4638.htm
44. Punnonen K, Irjala K, Rajamäki A. Serum transferrin receptor and its ratio to serum ferritin in the diagnosis of iron deficiency. Blood [Internet]. 1997 Feb 1; 89(3): 1052-7. Available from: http://www.ncbi.nlm.nih.gov/ pubmed/9028338

45. Guyatt GH, Oxman AD, Ali M, Willan A, Mcllroy W, Patterson C. Laboratory diagnosis of iron-deficiency anemia: an overview. J Gen Intern Med [Internet]. 7(2): 145-53. Available from: http://www.ncbi.nlm.nih.gov/ pubmed/1487761

46. Jindaratn Chaipokam, Thanyaphong Na Nakorn, Rojnuckarin Ponlapat. Diagnostic accuracy of reticulocyte hemoglobin content in Thai patients with microcytic red cells as a test for iron deficiency anemia. Asian Biomed 2016; 10: 31-7.

47. Balci Y, Akpinar F, Polat A, Uzun U, Ergin A. Evaluation of Reticulocyte Parameters in Iron Deficiency, Vitamin B12 Deficiency and Mixed Anemia. Clin Lab [Internet]. 2016; 62(03/2016). Available from: http://www.clinlab-publications.com/article/2043

48. Ceylan C, Miskioğlu M, Colak H, Kiliççioğlu B, Ozdemir $\mathrm{E}$. Evaluation of reticulocyte parameters in iron deficiency, vitamin B 12 deficiency and beta-thalassemia minor patients. Int J Lab Hematol [Internet]. 2007 Oct; 29(5): 327-34. Available from: http://doi.wiley.com/ 10.1111/j.1365-2257.2006.00872.x

49. Cai J, Wu M, Ren J, Du Y, Long Z, Li G, et al. Evaluation of the Efficiency of the Reticulocyte Hemoglobin Content on Diagnosis for Iron Deficiency Anemia in Chinese Adults. Nutrients [Internet]. 2017 May 2; 9(5): 450. Available from: http://www.mdpi.com/2072$6643 / 9 / 5 / 450$

50. Luo D, Chen Y, Wu W, Zhang F, Xu J, Cui W, et al. Reticulocyte hemoglobin content in the diagnosis of iron deficiency in Chinese pre-menopausal women. Chin Med J (Engl) [Internet]. 2007 Jun 5; 120(11): 1010-2. Available from: http://www.ncbi.nlm.nih.gov/ pubmed/17624271

51. Dinh NH, Cheanh Beaupha SM, Tran LTA. The validity of reticulocyte hemoglobin content and percentage of hypochromic red blood cells for screening iron-deficiency anemia among patients with end-stage renal disease: a retrospective analysis. BMC Nephrol [Internet]. 2020 Dec 22; 21(1):142. Available from: https://bmcnephrol.biomedcentral.com/articles/10.1186/s12882 $-020-01796-8$

52. Ageeli AA, AIGahtani FH, Alsaeed AH. Reticulocyte Hemoglobin Content and Iron Deficiency: A Retrospective Study in Adults. Genet Test Mol Biomarkers [Internet]. 2013 Apr; 17(4): 278-83. Available from: http://www.liebertpub.com/doi/10.1089/gtmb.2012. 0337

53. Buttarello M, Pajola R, Novello E, Mezzapelle G, Plebani M. Evaluation of the hypochromic erythrocyte and reticulocyte hemoglobin content provided by the Sysmex XE-5000 analyzer in diagnosis of iron deficiency erythropoiesis. Clin Chem Lab Med [Internet]. 2016 Jan 1; 54(12). Available from: https://www.degruyter. com/document/doi/10.1515/cclm-2016-0041/html

54. Toki Y, Ikuta K, Kawahara Y, Niizeki N, Kon M, Enomoto $M$, et al. Reticulocyte hemoglobin equivalent as a 
potential marker for diagnosis of iron deficiency. Int J Hematol [Internet]. 2017 Jul 15; 106(1): 116-25. Available from: http://link.springer.com/10.1007/ s12185-017-2212-6

55. Vázquez-López MA, López-Ruzafa E, Ibáñez-Alcalde $M$, Martín-González M, Bonillo-Perales A, LendínezMolinos F. The usefulness of reticulocyte haemoglobin content, serum transferrin receptor and the sTfR-ferritin index to identify iron deficiency in healthy children aged 1-16 years. Eur J Pediatr [Internet]. 2019 Jan 27; 178(1): 41-9. Available from: http://link.springer. $\mathrm{com} / 10.1007 / \mathrm{s} 00431-018-3257-0$

56. Malczewska-Lenczowska J, Orysiak J, Szczepańska B, Turowski D, Burkhard-Jagodzińska K, Gajewski J. Reticulocyte and erythrocyte hypochromia markers in detection of iron deficiency in adolescent female athletes. Biol Sport [Internet]. 2017; 2: 111-8. Available from: https://www.termedia.pl/doi/10.5114/biolsport. 2017.64584
57. Chinudomwong P, Binyasing A, Trongsakul R, Paisooksantivatana K. Diagnostic performance of reticulocyte hemoglobin equivalent in assessing the iron status. J Clin Lab Anal [Internet]. 2020 Jun 11;34(6). Available from: https://onlinelibrary.wiley.com/doi/ abs/10.1002/jcla.23225

58. Urrechaga E, Borque L, Escanero JF. Erythrocyte and reticulocyte parameters in iron deficiency and thalassemia. J Clin Lab Anal [Internet]. 2011; 25(3): 2238. Available from: http://doi.wiley.com/10.1002/ jcla.20462

59. Rungngu SLP, Wahani A, Mantik MFJ. Reticulocyte hemoglobin equivalent for diagnosing iron deficiency anemia in children. Paediatr Indones [Internet]. 2016 Jul 19; 56(2): 90. Available from: https://paediatricaindonesiana.org/index.php/paediatrica-indonesiana/article/view/115 